\section{artelogie}

\section{Artelogie}

Recherche sur les arts, le patrimoine et la littérature de l'Amérique latine

$15 \mid 2020$

Latin American networks: Synchronicities, Contacts and Divergences.

\title{
Mário Pedrosa, un parcours moderne 1900-1981
}

Jacques Leenhardt

\section{CpenEdition}

Journals

\section{Édition électronique}

URL : http://journals.openedition.org/artelogie/4929

DOI : $10.4000 /$ artelogie.4929

ISSN : 2115-6395

\section{Éditeur}

Association ESCAL

\section{Référence électronique}

Jacques Leenhardt, « Mário Pedrosa, un parcours moderne 1900-1981 », Artelogie [En ligne], 15 | 2020, mis en ligne le 08 avril 2020, consulté le 07 août 2020. URL : http://journals.openedition.org/artelogie/ 4929 ; DOI : https://doi.org/10.4000/artelogie.4929

Ce document a été généré automatiquement le 7 août 2020

Association ESCAL 


\title{
Mário Pedrosa, un parcours moderne 1900-1981
}

\author{
Jacques Leenhardt
}

\section{Introduction}

1 Né avec le siècle dans le Nordeste brésilien Mário Pedrosa appartient à une famille de notables cultivés, son père ayant été député et sénateur de l'Etat de Paraiba. Il s'éloignera rapidement de ce milieu dont la culture ne cessera cependant de l'accompagner au long d'une existence marquée par l'itinérance et l'engagement politique.

2 En 1914, il est envoyé étudier en Suisse mais la première guerre mondiale le contraint à revenir au Brésil où, une fois terminées ses études secondaires, il achève ses études de droit en 1923. Dès l'année suivante, il entreprend une activité de journalisme politique dans O Diário da Noite de São Paulo, débordant parfois vers la critique littéraire. Militant communiste, Mário Pedrosa devient responsable du Secours Rouge. Le Parti l'envoie se former en URSS mais la maladie le retient à Berlin où il reste finalement le temps d'une formation philosophique, sociologique et esthétique. 1928 le retrouve à Paris où il se lie avec plusieurs figures du groupe surréaliste, en particulier Benjamin Péret qui deviendra bientôt son beau-frère et Pierre Naville, qui facilitera son rapprochement avec l'opposition trotskyste. Exclu du РСB brésilien en 1929, Mário Pedrosa sera membre du Comité exécutif de la IV ${ }^{\mathrm{e}}$ Internationale, qu'il représentera à New-York en 1938 sous le pseudonyme de Lebrun. Ce séjour new-yorkais lui donnera l'occasion de travailler au MOMA. Après être rentré au Brésil pendant la dictature Vargas et avoir également rompu avec le bolchévisme, il est fait prisonnier et exilé aux Etats-Unis où il écrit son premier grand essai sur le peintre et fresquiste Cândido Portinari.

Ce bref résumé biographique permet de souligner l'importance que revêt le déracinement constant, social, géographique et intellectuel, dans la construction d'une conscience radicalement moderne chez Mário Pedrosa. L'exil signifie ici avant tout une 
distance prise à l'égard des conforts intellectuels et matériels, une façon parfois douloureuse d'aiguiser sa vision critique fondée sur une lecture marxienne du monde.

Après la guerre et la fin de l'Estado Novo, sans jamais s'éloigner cependant de l'arène politique, Mário Pedrosa se consacre de plus en plus à ses recherches et interventions dans le doáaine artistique. Il travaille comme critique dans plusieurs journaux (Correo da Manha (1945-1951), Estado de São Paulo (1951-1956), Jornal do Brasil (1957-1961), entre autres, et publie un essai fondateur sur l'œuvre de Alexandre Calder (1948). C'est l'époque où il présente sa thèse de doctorat: Da Natureza Afetiva da Forma na Obra de Arte, 1949 (De la nature affective de la forme dans l'œuvre d'art) où s'exprime une manière de phénoménologie de la perception esthétique qu'il a élaborée à partir des théories de la Gestalt de Wertheimer et Koffka auxquelles il s'était familiarisé durant ses années berlinoises. ${ }^{1}$ Mais en même temps qu'il développe une réflexion théorique sur la sensibilité aux foráes, Mário Pedrosa accompagne un groupe de jeunes artistes qui se réunit autour de l'Hospital Psiquiátrico do Engenho de Dentro ${ }^{2}$ localisé dans la banlieue de Rio de Janeiro. C'est une institution dirigée à l'époque par Nise da Silveira, une psychiatre jungienne qui a plus confiance dans les thérapies comportementales que dans la camisole chimique dans laquelle on enferme en général les schizophrènes accueillis dans l'hôpital qu'elle dirige. Dans ce climat intellectuellement très ouvert, un jeune artiste, Almir Mavignier, responsable de la «thérapie occupationnelle », a monté, en accord avec la directrice, un atelier de peinture destiné aux patients qu'il aide à se familiariser avec les objets de la peinture : le tableau, les couleurs, les brosses. L'activité picturale des malades se développe si bien que déjà Mavignier y voit des "artistes " fascinants par leur manière de composer leurs «tableaux » en dehors de toutes les lois du genre. C'est cette activité «sauvage » qui va séduire et interroger d'autres jeunes artistes, comme Yvan Serpa et Abraham Palatnik, mais aussi Mário Pedrosa qui y trouve un écho à ses préoccupations pour la forme telles que la psychologie de la Gestalt les avait aiguisées en lui.

Glaucia Villas Bôas a bien montré ${ }^{3}$ comment le travail intellectuel et le désir d'intervention dans le champ artistique, qui caractérisent Mário Pedrosa, se conjuguent dans cette expérience où les cadres traditionnels de la sensibilité sont remis en cause. Si on demande, ajoute-t-elle, les causes du surgissement de l'art concret à Rio dans les années 40, le rôle essentiel que joua l'Ateliê do Engenho de Dentro, l'apprentissage dont il fut le théâtre, les échanges intellectuels et sensibles qui s'y déroulèrent, apparaissent à l'évidence comme la condition d'émergence du programme concrétiste. Mário Pedrosa se révèle donc comme l'un des animateurs du virage concrétiste des artistes brésilien, qu'il soutiendra tout au long de leur développement.

6 Nise da Silveira le nota à l'époque : «les critiques d'art furent plus attentifs aux productions plastiques des schizophrènes que les psychiatres brésiliens ». ${ }^{4} \mathrm{Chez}$ Mário Pedrosa l'intérêt était vif pour ce qu'on appelait alors "l'art des fous", ou ce qu'on appellera plus tard «l'art brut», pour ces productions qui se développaient en dehors du cadre institutionnel de l'art. Hans Prinzhorn, un médecin suisse, avait été un des premiers collectionneurs et analystes de ces productions jugées marginales. Pour Mário Pedrosa au contraire, ces œuvres ouvraient directement une fenêtre sur les fondements anthropologiques, - et pas seulement culturels, - de l'expression plastique. Il avait rencontré cette même curiosité pour l'exercice incontrôlé de la créativité chez André Breton, une expression antérieure à toute aliénation que le théoricien du surréalisme avait formulée de façon concise: «l'œil existe à l'état sauvage ». C'était la phrase 
emblématique de cette préoccupation sur laquelle s'ouvrait Le surréalisme et la peinture, (1928).

7 L'effort de Mario Pedrosa pour donner un statut à l'art concret, puis néo-concret, fait partie d'une vision plus large qui englobe une volonté militante internationaliste s'opposant au repli nationaliste et folkloriste qui avait marqué certains moments du modernisme brésilien des années 30. Après guerre, cette bataille va déboucher sur un "projet constructif brésilien» où se manifeste la volonté de donner une place singulière au Brésil dans le concert des nations. Avec les années 50 et la présidence de Jucelino Kubitchek, cette dynamique trouvera un élan proprement national.

8 Il faut noter que le parti pris de Pedrosa en faveur du courant concrétiste le mettait en porte à faux avec la plus grande partie des forces de gauche de l'époque, notamment le parti communiste, qui plaidait pour un art «réaliste». Le critique avait bien connu cette dimension culturelle du mouvement révolutionnaire. Il avait lui-même, en 1932, développé une analyse des gravures de l'artiste socialiste allemande Käthe Kollwitz, qui sous sa plume s'inscrivait dans le droit fil du matérialisme historique. Il montrait comment le processus de rationalisation industrielle, abandonné à la logique aveugle du capital, avait conduit à une autonomie presque totale de la machine à l'égard des êtres humains, conduisant à une forme moderne d'aliénation. Les figures dessinées par Käthe Kollwitz lui apparaissent dès lors comme un antidote et une protestation.

9 L'inscription de ce texte dans le contexte militant de l'époque ne fait pas de doute. Il ne faudrait toutefois pas oublier que, à l'instar de Trotski, Mário Pedrosa ne pense pas que seul le prolétariat et l'art prolétarien sont appelés à contribuer à la révolution en cours. D'autres forces sont également au travail dans cet avant-guerre bouleversé et le rôle du critique d'art consiste à leur donner de la visibilité. Le critique combat alors sur plusieurs fronts: contre certains modernistes brésiliens, tentés par un retour aux traditions nationales, voire au folklore, mais aussi contre le diktat stalinien du réalisme socialiste, réducteur et rétrograde. Il mène un combat difficile dans ce contexte en faveur des formes pures du concrétisme et de son esprit constructiviste. C'est sur elles également qu'il s'appuiera dans un autre combat qu'il sera amené à livrer contre le subjectivisme de l'abstraction informelle et lyrique. Faisant une grande confiance à la sensibilité, Mário Pedrosa construit ses batailles sur les œuvres des artistes plutôt que sur des a priori théoriques. Ses argumentations sont des descriptions d'œuvres, toujours riches de ce savoir empirique que lui a donné la fréquentation de la pensée phénoménologique.

10 Au sortir de la guerre, Cândido Portinari est devenu le peintre emblématique de l'art engagé, la figure de proue de la gauche révolutionnaire brésilienne. Passé maitre dans l'art de la fresque, il s'inscrit dans la continuité symbolique et politique des muralistes mexicains. Toujours méfiant à l'égard de la figuration, plus encore lorsqu'elle se révèle didactique, Mário Pedrosa apprécie cependant les fresques que Portinari a produites en 1942 à Washington. Il y voit l'expression d'une liberté d'allure et même d'un « antinaturalisme » qui ne saurait lui déplaire. Il est cependant convaincu qu'il faut tourner la page de l'art figuratif et c'est ainsi qu'il va se trouver confronté à nouveau à l'œuvre de Portinari, au moment de devoir évaluer la fresque que l'artiste a dédiée au mouvement de l'Inconfidença (1949).

11 Développant une analyse serrée du panneau représentant le héros Tiradentes, Pedrosa néglige l'anecdote figurative, toute chargée de nobles sentiments, pour se concentrer exclusivement sur les éléments plastiques de l'œuvre. Il met ainsi en évidence, et en 
procès, l'inadaptation des moyens picturaux à la dimension monumentale du panneau $(17,70 \mathrm{~m} . \mathrm{x} 3,09 \mathrm{~m}$.). Il s'en prend à la conception même de cette peinture murale, héroïque et historique qui dénote une vision du monde dépassée et sans pertinence pour l'époque actuelle. Il souligne que l'omniprésence des détails, si caractéristique de la figuration réaliste entre en contradiction avec le gigantisme de l'œuvre. De ce fait, le spectateur ne parvient pas à saisir l'ensemble de la scène d'un seul coup d'œil et donc en manque la signification. Au terme de son analyse, Mário Pedrosa peut conclure : les transformations subies par le monde exigent un art nouveau, plus universel. Après le séisme du cubisme, l'abstraction constructive lui semble la seule voie permettant de dépasser une figuration compromise avec la réalité de l'exploitation dans le monde capitaliste contemporain. Un tel argumentaire constitue évidemment une critique dévastatrice du réalisme socialiste défendu alors par le Parti communiste brésilien.

L'art doit accompagner le processus social, mais cet accompagnement ne saurait se limiter à une simple illustration qui serait inévitablement secondaire et inessentielle. S'il doit jouer un rôle fondamental, l'art devra contribuer de manière spécifique à la construction du processus social. Ce sera alors par ses caractéristiques cognitives, par sa manière nouvelle de faire percevoir la réalité, qu'il démontrera sa capacité à transformer, et non à docilement reproduire, cette réalité. ${ }^{5}$

La critique d'art de Mário Pedrosa est une discipline de la pensée qui s'attache aux qualités techniques et objectives de la fabrication. L'artiste est à la fois, penseur et artisan, toujours aux prises avec la matérialité de ses instruments et de ses supports. La défense de l'œuvre de Alberto Volpi par Pedrosa en donne un bon exemple. Beaucoup de critiques de l'époque jugeaient l'œuvre de Volpi bien inférieure aux maîtres du moment qu'étaient Portinari, Guignard, Di Cavalcanti ou Segall. Sans doute paraissaitelle à leurs yeux trop simple. Or, pour Mário Pedrosa, c'est justement dans sa capacité à réaliser la synthèse des formes géométriques simples de la peinture moderne et du lyrisme des façades des maisons populaires, que la peinture de Volpi constitue un langage pictural d'actualité qu'il ne craint pas d'appeler « un événement artistique de premier ordre ». On voit dans cette appréciation du critique, le souci de libérer la peinture brésilienne de son écrasante dépendance par rapport à l'art européen, et français en particulier. La simplicité de Volpi résonne pour lui comme un cri d'indépendance en même temps que les formes simples qu'elle met en œuvre renvoient à la source pure de l'artisanat populaire. Pedrosa trouvait ainsi le moyen, à travers la peinture de Volpi, de magnifier une source architecturale populaire et brésilienne sans tomber dans le folklore et les relents de nationalisme si fréquents à l'époque. C'était aussi une manière de rendre caduques les efforts post-cubistes de nombreux artistes qu'il avait en horreur.

\section{Brasilia et le Congrès AICA 1959}

Il faut bien voir que la liberté de pensée Mário Pedrosa repose sur le constat que la culture brésilienne a été dévoyée par les importations constantes qu'elle a faites en puisant dans le bagage de l'hégémonie européenne et étasunienne. Il combat cette dépendance qui empêche que la réalité nationale brésilienne trouve ses solutions expressives propres. Il en est convaincu : l'heure est à décolonisation non seulement du pays économique mais aussi de sa culture. À l'instar des avant-gardes européennes qui voulaient brûler les musées, le Brésil doit affirmer sa propre modernité en rejetant, ou 
du moins relativisant, les formes artistiques prises par la modernité des « autres » car ces influences, nées dans la logique de situations différentes de celle du Brésil, finissent par créer une dépendance alors même qu'on croit se libérer en les accueillant.

Ainsi le rejet du réalisme, ou plutôt l'abandon du " préjugé réaliste ", tient chez Mário Pedrosa à la conviction qu'il s'agit d'une tradition européenne, dont par exemple l'art des indiens autochtones est totalement indemne. S'affirmer comme artiste brésilien implique donc de se libérer de ce "préjugé réaliste ». Ce n'est sans doute pas un hasard si cette attitude se développe chez lui de manière résolue dans les années d'après guerre, alors qu'il est revenu au Brésil après un exil de dix ans tout chargé d'une connaissance du monde d'une ampleur peu fréquente à l'époque. ${ }^{6}$ Celle-ci constitue le cadre intellectuel, idéologique et politique qui permet de comprendre l'organisation dans laquelle il se lance du Congrès International extraordinaire de l'AICA en 1959 à Brasilia.

16 Avec l'aide de Oscar Niemeyer, principal architecte de la nouvelle capitale Brasilia, Mario Pedrosa convoque la crème de la critique internationale dans le domaine de l'architecture, de l'urbanisme et des arts. Il s'agit de lui faire découvrir la réalisation en cours de la nouvelle capitale du Brésil. L'affaire a une dimension si évidemment politique que le Congrès est inauguré par le Président de la République, Jucelino Kubitschek, devant un parterre qui rassemble, parmi une centaine d'invités, Will Grohman, Eero Saarinen, John Entenza, Jean Leymarie, Stamos Papadaki, André Bloch, Charlotte Perriand, Jean Prouvé, André Wogenscky, William Holford, Bruno Alfieri, Giulio Carlo Argan, Meyer Schapiro, Gillo Dorfles, Alberto Sartoris et Bruno Zevi. ${ }^{7} \mathrm{Le}$ thème, Ville nouvelle-synthèse des arts, était clairement destiné à faire reconnaître par l'intelligentsia internationale, l'identité artistique nouvelle du Brésil. Les trois jours passés à Brasilia dans la poussière du chantier furent suivis par trois jours à São Paulo pour l'inauguration de la Biennale et autant à Rio de Janeiro, programme qui permettait de joindre à l'invention architecturale et urbanistique de la capitale à venir la production artistique des artistes brésiliens les plus modernes - notamment concrétistes et néo-concrétistes - mais également la production d'art populaire du Nordeste présentée par l'architecte Lina Bo Bardi dans son exposition Bahia no Ibirapuera.

17 La problématique Ville nouvelle-synthèse des arts s'inscrit dans un dispositif général dont l'origine pourrait bien être le texte séminal de Mário Pedrosa sur Calder (1944), rédigé alors qu'il résidait encore à New-York. L'œuvre de Calder y apparait comme la pierre de touche permettant de comprendre l'abandon de la fascination réaliste et son origine dans la modernité industrielle, celle que célébrait la Tour Eiffel (1914) de Delaunay. En délaissant le corps humain et sa symétrie, auquel sont attachées les problématiques académiques de la sculpture - le volume, la surface, le modelé - Calder ne conserve, note Mário Pedrosa, que les possibilités du matériau. C'est en privilégiant la dynamique du déséquilibre qu'il est vraiment moderne. Plutôt qu'à l'homme de Vitruve, l'esthétique de Calder renvoie à l'arbre, à son déséquilibre entre un tronc massif et stable et son feuillage sensible au vent et capteur d'incertitudes changeantes. Il faut noter toutefois que cette modernité n'est pas celle de la technologie : Calder est peutêtre celui qui fait l'usage le moins technique de la technique elle-même. Pour cette raison Pedrosa peut le tirer vers un univers symbolique où l'homo faber bascule vers les possibilités infinies - et infiniment surprenantes - de ce qu'offre la nature. Dans cette métaphore de l'arbre, Pedrosa retrouve ce qu'il admirait déjà chez Cézanne, une forte 
construction contrebalancée par le pouvoir, fragile mais insistant, de la "petite sensation ", selon l'expression du peintre.

Calder prolonge cette lignée qui s'ouvre sur la transformation, voir la révolution, de la sensibilité. En construisant ses machines qui ne servent à rien, l'artiste dépasse l'héritage fonctionnel du machinisme : il offre à la modernité de nouveaux paradigmes de pensée et de sensation. Or c'est à travers l'exploration de ces fonctions cognitives, dont la Gestalt étudie le champ, que l'art remplit pleinement sa fonction sociale de transformation de la perception et de la sensibilité. L'esthétique assume donc une fonction éthique d'innovation sociale dont Brasilia fournit un des exemples les plus imposants.

Mário Pedrosa n'est pas aveugle sur les contradictions qui se manifestent dans le projet de cette capitale implantée artificiellement au centre géographique du pays. Il sait que les cultures organiques de l'Europe historique se sont faites dans des cités reliées entre elles par des routes et des voies de chemin de fer. Il n'ignore pas non plus que faire reposer sur la seule connexion aérienne le lien avec le reste du pays qu'elle est censée organiser est, pour une capitale, un pari très audacieux. Mais, et en cela il est cohérent, il rappelle que le Brésil n'est pas né naturellement, que la modernité lui a été en quelque sorte imposée dès sa naissance par la dépendance dans laquelle il a grandi. Aussi, de même que le Brésil a été jeté dans la modernité artificielle par la colonisation, de même Brasilia sera une civilisation oasis. Tel est son destin et dans cette situation difficile, il faut faire le pari radical de la modernité.

En attendant, le projet de modernisation du Brésil se trouve politiquement transformé par le coup d'Etat du 31 mars 1964. Le renversement de la II République était préparé de longue date par les militaires brésiliens, avec à leur tête le général Castelo Branco, proche de l'armée nord-américaine et bénéficiant de l'appui de la CIA. Le Président João Goulart est renversé au terme d'une longue période d'instabilité sociale, d'hyperinflation et de montée d'un anticommunisme violent prenant prétexte de l'alignement de Cuba sur l'Union soviétique.

\section{Mario Pedrosa et le Musée de la Solidarité Salvador Allende (MSSA)}

Invité par Miguel Rojas-Mix à participer aux travaux de l'Institut d'Histoire de l'art latino-américain de l'Université du Chili alors qu'il était exilé dans ce pays par la dictature brésilienne, Pedrosa met en œuvre, avec le soutien du Président Salvador Allende, le montage d'un Musée de la solidarité. Il a alors 71 ans.

Il semble qu'il y ait eu au moins deux projets de Musée, celui conçu par Miguel RojasMix, dans une perspective continentale latino-américaine et anti-US et celui de Pedrosa, plus international et plus expérimental. Fortement politiques l'un comme l'autre, les deux concepts relèvent cependant d'ambitions différentes. Rojas-Mix, comme la plupart des intellectuels des pays hispanophones, prend l'identité continentale en train de s'affirmer comme horizon, dont Cuba est à l'époque le catalyseur. Appartenant à un pays qui s'est toujours pensé lui-même comme un continent, et peu enclin à se fondre dans le continent latino-américain, Mário Pedrosa imagine le futur musée comme le résultat de la convergence de la modernité de l'art et de la modernité du projet politique de Salvador Allende. 

général Augusto Pinochet assassine Salvador Allende et renverse l'Unité populaire au pouvoir. Mário Pedrosa se réfugie à l'Ambassade du Mexique d'où il charge Daisy Peccinini d'organiser l'exfiltration des documents du Musée. Il poursuivra ensuite la lutte depuis le Mexique, transformant le Musée de la Solidarité Salvador Allende en un «musée itinérant, symbole de résistance ». Prenant exemple sur l'exil emblématique du Guernica de Picasso, ce musée de la générosité à l'égard du peuple chilien ne devra retourner dans le pays auquel il est destiné qu'avec le retour de la démocratie. De fait, une nouvelle histoire, itinérante et internationale, commençait pour ce musée singulier dont Mario Pedrosa, détenteur désormais d'un passeport de réfugié politique français, poursuivra la constitution et l'enrichissement en favorisant son développement au Mexique, en Espagne, en Suède, en Pologne, à Cuba et en France. C'est alors que je l'ai connu.

De nombreux Chiliens avaient émigré en cet automne 1973. Artistes, critiques, directeurs de musée, ils étaient nombreux à s'être retrouvés à Paris où se forme alors un noyau de soutien aux chiliens exilés et une équipe de bénévoles dénonçant la brutalité de la nouvelle dictature dans un livre : Le livre noir de la répression au Chili qui paraîtra quelques mois plus tard.

28 C'est dans ce cadre que je commence à collaborer à l'automne 1973, d'abord avec le groupe du Livre noir autour de Julio Cortázar, puis avec le Comité du Musée en exil qui 
se avec forme autour de Miria Contreras, qui avait été secrétaire de Allende, Carmen Waugh et des trois représentants des partis de l'Unité populaire, qui avait soutenu Allende : José Balmes pour le PC, Miguel Rojas-Mix pour la démocratie chrétienne et Pedro Miras pour le parti socialiste.

Mário Pedrosa, continue à suivre le développement du Musée mais l'ampleur prise par le projet $d u$ fait du coup d'état a en quelque manière dissout le projet originel qu'il avait porté. Celui-ci était davantage lié à une politique de la transformation des catégories mentales grâce à la diffusion des œuvres d'art qu'à une politique de soutien aux mouvements politiques de la résistance contre la dictature.

La signification donnée au mot "résistance " s'avère déterminante, soit qu'on la conçoive, comme Pedrosa lui-même, d'abord dans le domaine artistique et par rapport à ses implications dans la transformation socio-politique, soit qu'on y voie un instrument de lutte devant servir immédiatement la cause politique. C'est ainsi que les options des uns et des autres divergèrent à diverses reprises lorsque se posa par exemple la question de savoir si le Musée pouvait - ou éventuellement même devait vendre certaines œuvres d'art . Les uns considéraient que le fait que les œuvres avaient été données pour la "résistance » justifiait et légitimait qu'on les vende pour soutenir les mouvements populaires de résistance à la dictature, d'autres pensaient que c'était le musée qui était un acte de résistance. Ainsi passèrent les années jusqu'à la chute de la dictature et la recréation-création du Musée International de la Solidarité Salvador Allende à Santiago.

\section{Conclusion}

L'ordre d'incarcération de Mário Pedrosa au Brésil ayant été annulé en 1977, le critique d'art retourna dans son pays pour y réaliser divers projets comme l'exposition consacrée à l'art indigène Alegria de viver, alegria de criar, qui devait être accueillie au Musée d'Art Moderne de Rio de Janeiro. Toutefois le MAM brûla accidentellement et l'exposition n'eut pas lieu. Cette occasion manquée permit à Mário Pedrosa de proposer une nouvelle conception, voire une nouvelle stratégie muséale ambitieuse, embrassant le MAM dans un dispositif plus large où il aurait été articulé à quatre autres structures autonomes reliées par un projet unique : le Musée de l'Indien, le Musée de l'art vierge (derrière ce titre il faut voir le musée de l'inconscient auquel s'était attachée Nise da Silveira), le Musée du Noir et le Musée des arts populaires.

Ce projet n'eut bien évidemment pas de suite institutionnelle. Il est cependant absolument emblématique de la réflexion que mène Mário Pedrosa depuis les années 40 : retrouver les racines anthropologiques de l'art, sans confinement artificiel, donner tout son espace à cette spontanéité humaine qui s'exprime dans différentes formes d'art depuis quarante millénaires tout en renouvelant constamment ses formes. On comprend à quel point ce programme repose sur le rôle cardinal que joue la théorie de la Gestalt dans l'esprit de Mário Pedrosa en tant que fondement d'une psychologie globale de la perception visuelle. L'articulation des cinq musées dans un projet unique constitue, pour Mário Pedrosa, le compromis qu'il est prêt à passer avec la division institutionnelle des champs et des disciplines. Ce programme qui implique une redéfinition de la notion même de musée repose sur le fait que toute perception visuelle met en jeu des formes et des modes de vision - qu'on pourrait appeler artistiques - de telle sorte qu'il est nécessaire de conclure que toute pensée rationnelle 
est en même temps intuition, que toute perception est en même temps pensée et que toute observation est en même temps invention.

En proposant d'articuler ces diverses institutions dans un programme global, Mário Pedrosa visait à décloisonner les champs épistémiques, à élargir le concept et le territoire de l'art, à connecter l'art dit primitif aux autres formes, sages ou folles, d'expression de la sensibilité, à mettre le spectateur dans une position active au sein du dispositif. Que cet universalisme fondé sur les structures anthropologiques de la perception constitue également un programme politique, c'est ce que Mário Pedrosa a tenté de prouver dans les différentes institutions muséales dont il a eu la responsabilité. C'est cela qui constitue son legs le plus innovant et fondateur.

\section{NOTES}

1. Une série d'essais critiques, une anthologie de textes de l'auteur, une chronologie ainsi qu'une bibliographie de Mário Pedrosa figurent, en langue espagnole, dans le catalogue de l'exposition qui lui a été consacrée par le Centro de Arte Reina Sofía sous le titre: Mário Pedrosa De la Naturaleza afectiva de la forma, Madrid, Museo Nacional Centro de Arte Reina Sofía, 2017. Traduits cette fois en anglais, on trouve une autre anthologie de textes ainsi que d'autres essais critiques sur son œuvre dans: Mário Pedrosa, Primary Documents, Glória Ferreira and Paulo Herkenhoff (Eds.), New-York, the Museum of Modern Art, 2015

2. Cet atelier réunit à Rio de Janeiro, de 1946 à 1951, artistes, critiques d'art, médecins et patients du Centre psychiatrique national Pedro II.

3. Glaucia Villas Bôas, « A estética da conversão, $O$ ateliê do Engenho de Dentro e a arte concreta carioca

(1946-1951) » in Tempo Brasileiro, USP, São Paulo, vol. 20, N 2, 2008

4. Nise da Silveira, Imagens do inconsciente, Rio de Janeiro, Alhambra, 1981 p. 14, cité par Glaucia Villas Bôas, op cit. p. 206

5. Il serait intéressant de comparer la critique de la peinture de Portinari par Pedrosa et celle de Fougeron par Aragon dans un article au titre programmatique : "Pour un réalisme véritable » paru dans Les Lettres françaises, $\mathrm{N}^{\circ}$ 490, du 12 novembre 1953.

6. Voir la préface de Otília Arantes à : Mário Pedrosa, Textos escolhidos Acadêmicos e modernos, Otília Arantes org. São Paulo, Edusp, 1998

7. Eduardo Pierrotti Rosetti note que durant cette année 1959, de très nombreux visiteurs de qualité furent ainsi invités, dont les noms vont de la Duchesse de Kent à Fidel Castro et d'André Malraux à Golda Meir et au Président d'Indonésie, «Brasília, 1959: a cidade em obras e o Congresso Internacional Extraordinário dos Críticos de Arte" in Arquitextos N 111.03, São Paulo, Vitruvius, 10e année, août 2009 


\section{RÉSUMÉS}

En proposant d'articuler diverses institutions dans un programme global, Mário Pedrosa visait à décloisonner les champs épistémiques, à élargir le concept et le territoire de l'art, à connecter l'art dit primitif aux autres formes, sages ou folles, d'expression de la sensibilité, à mettre le spectateur dans une position active au sein du dispositif. Que cet universalisme fondé sur les structures anthropologiques de la perception constitue également un programme politique, c'est ce que tentera de montrer cet article analysant les différentes institutions muséales dont il a eu la responsabilité.

Proponiendo articular varias instituciones en un programa global, Mário Pedrosa tuvo como objetivo descompartimentalizar los dominios epistémicos, ampliar el concepto y el territorio del arte, conectar el arte primitivo con otras formas, sabias o dementes, de expresión de la sensibilidad, para poner al espectador en una posición activa dentro del dispositivo. Este artículo intentará mostrar que el universalismo defendido por el critico latino-americano basado en las estructuras antropológicas de la percepción, también constituye un programa político, en particular en el análisis de las diferentes instituciones y museos de las cuales Pedrosa tuvo la responsabilidad.

\section{INDEX}

Mots-clés : Mário Pedrosa, musées, structures anthropologiques de la perception, art et politique.

Palabras claves : Mário Pedrosa, museos, estructuras antropológicas de la percepción, arte y política.

\section{AUTEUR \\ JACQUES LEENHARDT}

EHESS 